\title{
Ontological Pluralism and Multi-Quantificational Ontology
}

\author{
Zbigniew Król $^{1}$ (D) Józef Lubacz ${ }^{1}$ (D)
}

Accepted: 6 July 2021 / Published online: 19 July 2021

(C) The Author(s) 2021

\begin{abstract}
This paper explores some variants and aspects of multi-quantificational criteria of existence, examining these in the context of the debate between monism and pluralism in analytical philosophy. Assuming familiarity with the findings to date (summarized in broad terms at the outset), we seek to apply to these the newly introduced concepts of "substitution" and "substitutional model". Possible applications of formal theories involving multiple types of existential quantifier are highlighted, together with their methods of construction. These considerations then lead to a thesis asserting the irrelevance of both multi-quantificational criteria and assumptions involving quantificational ontology to the debate between monism and pluralism in ontology. Many quantifiers cannot properly distinguish different modes of existence-as we aim to show by furnishing a general method for constructing counter-examples to any theory that assumes that different types of existential quantifier correspond to different modes of existence.
\end{abstract}

Keywords Ontological pluralism $\cdot$ Multi-quantificational criteria of existence $\cdot$ Modes of existence $\cdot$ Monism/Pluralism debate $\cdot$ Models $\cdot$ Model theory

\section{Introduction}

The present article aims to investigate multiple versions and aspects of multi-quantificational criteria of existence in the analytical debate concerning ontological pluralism. Its conclusion will be that in the context of theories employing multiple types of existential quantifier for ontological purposes, most of these are to be rejected. "Something is needed" is not equivalent to "something exists", and the purposes embodied in "our needs" are not themselves the same as "ways of being". Multiple quantifiers are unable to distinguish between modes of existence, in the same way as a single quantifier is unable to do so.

We shall call the sort of criteria aimed at distinguishing different modes (ways) of existence to which our theories are supposed to be committed multi-quantificational criteria of

Zbigniew Król

zbigkrol@wp.pl

Józef Lubacz

jozef.lubacz@pw.edu.pl

1 Faculty of Administration and Social Sciences, International Center for Formal Ontology, Warsaw University of Technology, Plac Politechniki 1, Main Building room 203, 00-661 Warsaw, Poland 
existence (M-QCE). In Section II, we indicate how, and for what mathematical and ontological reasons, theories with multiple different quantifiers may be constructed. The conclusions stemming from that analysis are then generalized in Section III, as a basis for our subsequent elaboration (in Section IV) of what, in practice, is entailed by the introduction of the notion of substitutional models central to our present considerations. The idea of such a model amounts to a certain generalization of the standard conceptual framework of model theory-one independent of its current application to M-QCE. Thus, in Section III, we formulate certain general theorems we take to be relevant-relevant, that is, in the context of a discussion of M-QCE whose goal is not only that of explicating the role of these substitutional models within quantificational ontology itself, but also that of indicating how they could be deployed in the construction of counter-examples to quantificational ontological pluralism. Next, we invoke Tarski's concept of interpretability as essential to a proper inquiry into substitutional models. Section IV, meanwhile, consists mainly in a more systematic presentation of the concept, and methods of construction, of substitutional models, and at this juncture we employ the models in question to address the validity of various different M-QCE options. Finally, Section V summarizes our main conclusions, and presents some additional considerations pertaining to possible versions of M-QCE.

The remarks that follow immediately below begin by sketching in general terms the background to the ensuing analysis. We should add that it was not feasible, for reasons of length, to deal directly with all versions of M-QCE as presented in the literature. We therefore limit ourselves to those basic threads pertaining to the issue that we think will suffice to ensure the comprehensibility of our essential lines of argument.

Existential quantification is only one of the many tools used in philosophical considerations of what there is. Quantification has become one of the main ways of approaching ontological questions of existence in science and philosophy, especially after Quine (1948, 1969). However, in this text we shall not be discussing Quine's monistic approach (except for some absolutely essential remarks), as almost everyone agrees that a single quantifier quantifies over all objects regardless of their mode of existence. A relatively new approach concerns the application of multiple quantifiers in the debate between monism and pluralism; cf. (Turner, 2008, 2012, 2013, 2018, 2020, McDaniel, 2009, Javier-Castellanos, 2019). Such theories are interesting for philosophers defending pluralism because, in their opinion, a multiplicity of existential quantifiers can indicate a plurality of modes of existence; see, e.g., (Turner, 2013, 2018, Spencer, 2012). In the following, we enter into this debate, proceeding on the assumption that pluralism amounts to the conviction that there are many ways of being.

Under a typical Quinean scenario with just one type of quantifier, existence seems to be univocal; cf. (Garbacz, 2019). If a (physical, formal) theory uses natural numbers, the numbers can be presumed to exist "in the same way" as electrons, atoms, electromagnetic waves and other physical/material objects postulated by the theory. Thus Quine's criterion implies that our physical theories are committed to accepting the existence of multiple (non-physical?) mathematical objects, such as numbers, geometrical lines, surfaces and points. ${ }^{1}$ This intuitively strange conviction is just one of many potential reasons to embrace pluralism. Even so, while declaring ourselves committed pluralists, we will not be addressing the arguments in favour of that position in this text. What we instead seek to show here is that the arguments referring to the use of theories with multiple quantifiers in ontology

\footnotetext{
1 At the same time, there have been many monistic attempts to eliminate such objects from physics; see, e.g., (Balaguer, 1996; Field, 1980).
} 
are flawed, because quantifiers are not able to correctly identify different ways of existing. With this in mind, we outline a general method for the constructing of counter-examples to a theory with multiple quantifiers-one that aims to correctly distinguish between these different ways. Regardless of how this distinction is made, we show-in our discussion, below, of substitutional theories and models-how a theory can be constructed in which the same quantifiers also operate on objects having a different mode of existence from the one assumed when defining them (i.e. objects "reserved", so to speak, for other quantifiers). If it is always possible to construct such a counter-example, then this means that quantificational pluralism is not able to distinguish correctly between different modes of existence.

In our view, analytical considerations pertaining to the use of multiple quantifiers to precisely capture different ways of existing descend into a vicious circle. On the one hand, the discussion is, for the most part, elaborated informally, so there are no obstacles to each participant understanding what "different modes of existence" amount to or grasping the rationale behind their adoption, while on the other, all the efforts of ontological pluralists are aimed at constructing, in the (distant?) future, a formalized theory with multiple different existential quantifiers, so they only seek to achieve this as a possibility. Our point is that the very idea of justifying the existence of different ways of existing by constructing a formal theory that makes it possible to talk about them is not justified, as philosophers can obviously speak rationally about modes of existence in the absence of any such theory. The premise of this discussion, then, is that the existence of such a theory constitutes an ontological proof of the existence of objects that exist in different ways. Thus, the theory may be said to entertain pretensions to-in a sense-creating reality. (In short, what actually exists in reality is regarded as being of little or no importance; instead, what matters is just what can be formulated, providing this can be done in a formally precise manner.)

At the same time, for the purposes of this text we shall simply assume that there are, indeed, theories with multiple quantifiers that indicate, in an exact way, different modes of existence. As we shall see, with the use of our substitutional models it is relatively easy to construct counter-examples to each and any such theory. Nevertheless, there are many different types of substitutional model, and it is rather difficult to find one general pattern to describe them. Thus, we shall only propose our method itself in intuitive terms, and it will be necessary in each case to arrive at a detailed and precise formal description of the required counter-example.

The substitutions and substitutional models we employ enables falsifying almost (cf. the last section) any possible kind of quantificational criterion of existence in respect of its value as a tool for pluralistic quantificational ontology. Moreover, this is so even when we take into consideration theories amounting to more than mere "notational variants" of the kind reducible to a monistic conception; cf. (Turner, 2012).

One of several possible general attitudes on whose basis "quantificational ontology" can be developed, further referred to as strong theoretical existence (STE), is the (analytical) conviction that that there is no possible way to discuss questions of existence at all if there is no formal theory in which one can express the existence of something ("ontological scientism"; cf., for instance, Quine's monistic holism). Thus, according to STE, the only way to cultivate a scientific ontology is by means of a formalization or translation of expressions from folk languages into their formal counterparts. This leads to the view that the only way to pursue ontology is to philosophize in terms directly connected with science. STE-as will be demonstrated-can be combined with different versions of Quine's quantificational criterion of existence (QCE) or M-QCE.

In such contexts, one can consider several versions of the quantificational criterion of existence, for instance: 
- Strong QCE: only theories containing irremovable formulae involving existential quantifiers are ontologically committed, and every formula (with or without parameters) or sentence containing existential quantifiers is ontologically committed. For this reason, Quine (1969) claims that theories with finite models (in which we can eliminate quantifiers by replacing them with certain finite formulae without quantifiers-e.g. certain conjunctions, or disjunctions) or complete ones (from which we can eliminate quantifiers) have no ontological commitments.

- Strong QCE+ : As above, except that (only or also) formulae possessing certain special ("elite", "generic", etc.) quantifiers are considered, such as will pick up on different ways of being in ontology, and only such theories are ontologically committed to objects of different modes of existence.

- Strong M-QCE: different types of existence demand that different quantifiers be employed, i.e. we can't have a classical theory with just one quantifier applied over ontologically different objects. Existential quantifiers are thus seen as furnishing an appropriate tool for describing different modes of existence.

- Weak M-QCE: ontological pluralism is possible iff it is possible to construct a theory with multiple different existential quantifiers, and the same object can appear in a theory with one existential quantifier and also in a theory with multiple (really) different existential quantifiers, where this means that different quantifiers can distinguish between different modes of existence, but do not have to do so automatically.

In Section V we mention some other possible sub-variants of the above, nevertheless we do not refer to all aspects of quantification-related issues covered in the literature-e.g., in (Turner, 2013; Janssen-Lauret, 2016; Menzel, 1990; Schwartz, 1993; Thibaut, 2013). ${ }^{2}$ In particular, as was already indicated, we do not devote special attention to such constraints that may be imposed on existential quantifiers as, for instance, Turner's "elite quantifiers" (2018).

As we shall see below, M-QCE fail to adequately identify the modes of existence of objects within theories: in a given theory, quantification occurs over the names of (formal) objects, and not over the modes of existence of the objects postulated by the theory. Moreover, M-QCE cannot adequately indicate what really exists according to a theory.

\section{The Rationale and Limitations of Multi-Quantificational Criteria of Existence}

We begin by constructing a formal theory which, although it assumes multiple existential quantifiers-indeed, however many one wishes-cannot satisfactorily differentiate modes of being. ${ }^{3}$ First of all, note that a classical theory with two different existential quantifiers will be inconsistent if (a) we assume the existential and general quantifiers to be interrelated along classical lines, and (b) there is only one general quantifier possible:

\footnotetext{
2 As well as these, some further sources relevant to this may be found, for instance (Eklund, 2014; Lavine, 2000; Marcus, 1972; McDaniel, 2009; Parsons, 1970; Prior, 1971; Putnam, 1971; van Inwagen, 2003).

3 We are talking about "theories" here, because they are ultimately the ones at stake, but the changes associated with the introduction of multiple quantifiers obviously concern mostly the logic of these theories.
} 


$$
\begin{aligned}
& \exists_{1} x \cdot F(x) \equiv \sim \forall x . \sim F(x) \\
& \exists_{2} x \cdot F(x) \equiv \sim \forall x . \sim F(x)
\end{aligned}
$$

thus

$$
\exists_{1} x . F(x) \equiv \exists_{2} x . F(x)
$$

Alleviating condition (b) would lead to us introducing two new symbols, say " $\forall$ " and " $\forall \mathbf{2}$ ", but this seems quite artificial, and is difficult interpret if their domains are not differentiated. ${ }^{4}$ There is, however, another possibility, illustrated by the following example.

Define, besides the classical universal and existential quantifiers, two new specific existential quantifiers: $\exists_{\mathbf{c}}$-to quantify over concrete objects, and $\exists_{\mathbf{a}}$-to quantify over abstract objects. Assume the following relations:

$$
\begin{gathered}
\exists x . F(x) \equiv \exists_{c} x \cdot F(x) \vee \exists_{a} x \cdot F(x) \\
\exists_{c} x \cdot F(x) \rightarrow \sim \exists_{a} x \cdot F(x) \\
\exists_{c} x \cdot F(x) \rightarrow \exists x \cdot F(x) \\
\exists_{a} x \cdot F(x) \rightarrow \exists x \cdot F(x) \\
F(a) \rightarrow \exists_{c} x \cdot F(x) \vee \exists_{a} x \cdot F(x)
\end{gathered}
$$

We can resign from condition (5), if we accept that some concrete objects can be abstract ones. Condition (4) can be modified as follows, providing we accept that our universe does not consist only of abstract and concrete objects:

$$
\exists_{c} x \cdot F(x) \vee \exists_{a} x \cdot F(x) \rightarrow \exists x \cdot F(x)
$$

In that case, we can also introduce another existential quantifier to modify condition (8). Obviously, the above method can also be generalized, allowing one to introduce as many quantifiers as one needs.

Conditions $(4,5,6,7,8)$ imply that the universe is a disjointed sum of two classes: $\mathbf{C}$, the class of concrete objects, and $\mathbf{A}$, the class of abstract ones. Hence, the theory being introduced is interpretable in Tarski's sense (cf. below), in a theory with restricted quantifiers:

$$
\begin{aligned}
& \exists_{c} x \cdot F(x) \equiv \exists x \in C \cdot F(x) \\
& \exists_{a} x \cdot F(x) \equiv \exists x \in A \cdot F(x)
\end{aligned}
$$

Obviously, subdomains $\mathbf{A}$ and $\mathbf{C}$ can be determined in many different ways in a model of our theory, without changing the type of the quantifiers. Thus, what counts as a concrete

\footnotetext{
${ }^{4}$ Different universal quantifiers are considered in (Turner, 2013), but this unfortunately lacks a satisfactory interpretation; the quantifiers introduced are, in fact, domain relative. Turner addresses this explicitly in (2012).
} 
or an abstract object does not depend on the existential quantifiers, but on specific predicates used in the theory, and on our choices. This is congruent with the usual practice of differentiating between hypothetical types of being: we say "d is an abstract object" and "e is a concrete object", where "d" and "e" are some constants.

It is worth noting at this point what Turner (2012, p. 419) has to say about this: "To be (in the abstract way) is to be needed as the value of a variable bound by ' $\exists_{\mathrm{a}}$ '; to be (in the concrete way) is to be needed as the value of a variable bound by ' $\exists_{\mathbf{c}}$ '." Thus, the type of quantifier will be determined by the formula and its domain, and there will be appropriate ways to determine these, beyond the mere use of different symbols. This is precisely because, as Turner himself rightly notes, ' $\exists_{\mathbf{a}}$ ' and ' $\exists_{\mathbf{c}}$ ' are only symbols from a formal point of view, that he is led to invoke special ontological quantifiers in (2018).

Let us, in every formula of our theory, replace $\exists_{\mathbf{c}}$ with $\exists_{\mathbf{a}}$ and vice versa. Since the mode of existence depends only on the kind of existential quantifier involved, and is independent of any other formal properties, the abstract objects will become concrete ones, the concrete ones abstract. Therefore, the type of existential quantifier-in this narrow sense-is not responsible for the mode of existence of the objects. Thus, quantifiers constructed for an ontological purpose really should be special: i.e. unchangeable and asymmetrical.

The questions we may then ask is this: what, in that case, could be the purpose served by our adopting a theory like the one just introduced (or, indeed, any multi-quantificational theory)? Does ontological commitment itself furnish the only reason to do so? ${ }^{5}$

In order to answer these questions, let us consider two different first-order theories, such as ZFC (Zermelo-Fraenkel set theory with the axiom of choice) and PA (Peano arithmetic), and the possibility of unifying them in one syntactic consistent formal theory. Imagine that we were to group together the axioms of these theories. Such a theory would not be consistent, so first of all, its relational symbols would have to be changed in order that there would be no relational symbols in common between ZFC and PA (including the relation of identity "="). ${ }^{6}$ This would not suffice, though: the theory would still be inconsistent, because of the quantifiers. The next step would be to introduce specific existential quantifiers, for instance as described above, such as $\exists_{\mathrm{c}}$ in ZFC and $\exists_{\mathrm{a}}$ in PA, and invoke conditions $(4,5,6,7,8)$ as mentioned above. ${ }^{7}$ It can be straightforwardly demonstrated that the resulting unified theory would then be consistent (iff ZFC and PA are consistent).

Of course, this kind of theory-unification procedure is not the only possible one. In particular, procedures which use only one type of existential quantifier are also possible: on this point, it will suffice to take cognizance of theories with restricted quantifiers or substitution procedures as described in the next section. The above illustration also indicates the feasibility and purpose of introducing more than two types of existential quantifier. On the other hand, the method of unifying even totally different formal theories using multiple types of quantifier in some respects lends support to M-QCE.

In the context of these considerations, it is important to emphasise that there are many possible theories referring to multiple modes of existence which nevertheless use only one existential quantifier. For instance, there is an obvious possibility of constructing a relevant

\footnotetext{
${ }^{5}$ Until now, with the exception of many-sorted logics and restricted quantifiers, there has been no requirement to use multiple different quantifiers in science or mathematics. Below, we indicate some further mathematical and logical reasons for using multiple existential quantifiers in mathematics.

${ }^{6}$ Obviously other symbols, e.g. logical connectives, will remain the same in both theories.

7 Invoking conditions $(4,5,6,7,8)$ would enable us to add some new theorems not contained in the sum of the theses of the two theories.
} 
concept calculus, ${ }^{8}$ which would formalize our intuitive, informal concepts of different modes of existence. From this point of view, in the context of formal ontology and, especially, ontology proper, the multi-quantificational theories are cumbersome and artificial. Note in particular that in the theory introduced above, there are three different existential quantifiers referring to just two different modes of existence.

\section{Some Generalization}

The observations made above can be generalized with the application of the following Theorem and its Corollary. In particular, a more general theory than that described by conditions $(4,5,6,7,8)$ can be constructed. Our conception of the constructions needed in the proofs of the theorem and its corollaries will be presented in the next section.

Let us first remind ourselves of the notion of the interpretability of a theory $\mathrm{T} 1$ in the theory T. There are many possible definitions of that notion, be they syntactical or semantical. Tarski defined the concept in a syntactical way in (1968, pp. 20-22); cf. also (Friedman, 2007, 2009, 2012).

From an intuitive point of view, T1 is interpretable in T2 when one is able to extend T2 with definitions of functions and relations of T1 using only means from T2. Thus, when T1 is interpretable in $\mathrm{T} 2$, one can speak about objects from $\mathrm{T} 1$ in $\mathrm{T} 2$ being enriched with the relevant translation of the relations and functions of $\mathrm{T} 1$ into the language of T2; such an enriched T2 will be named T. For instance, in three-dimensional Euclidean geometry one can consider two-dimensional plane geometry, while in ZFC we encounter von Neumann's definition of natural numbers, meaning that PA is interpretable in ZFC.

More strictly, assume that we have two theories, T1 and T2, that do not have common non-logical constants. We say that $\mathrm{T} 1$ is interpretable in $\mathrm{T}$ iff $\mathrm{T}$ is a common extension of $\mathrm{T} 1$ and $\mathrm{T} 2$ in such a way that:

(i) $\mathrm{T}$ contains $\mathrm{T} 1$ and every non-logical constant (i.e. constant, relation or function) of $\mathrm{T}$ is a non-logical constant of $\mathrm{T} 1$ or $\mathrm{T} 2$;

(ii) there is a recursive (iff there are infinitely many constants in T1) or finite (iff there is a finite set of constants in T1) set D of definitions of non-logical constants of T1 in terms of $\mathrm{T} 2$, meaning that each non-logical constant of $\mathrm{T} 2$ appears in only one place (in the definiendum) in $\mathrm{D}$;

(iii) every valid sentence of $\mathrm{T}$ is derivable in $\mathrm{T}$ from the set of sentences of T1 or D.

If there are common constants in $\mathrm{T} 1$ and $\mathrm{T} 2$, they must be renamed in order to give theories with disjoint sets of constants; in that scenario, a theory T1' will be obtained, and $\mathrm{T} 1$ will be said to be interpretable in $\mathrm{T} 2$ iff $\mathrm{T} 1$ ' is interpretable in $\mathrm{T} 2$ according to the above definition.

Theorem For every two consistent first-order (or any-other-order) theories, $T 1$ and T2, there is a consistent ${ }^{9}$ first-order (or higher-order) theory $T$ in which $T 1$ and $T 2$ are interpretable according to Tarski's concept of interpretability.

\footnotetext{
8 See, e.g., (Friedman, 2009, 2012), as well as other works of the same author dealing with concept calculi, accessible online at http://u.osu.edu/friedman.8/foundational-adventures/downloadable-manuscripts/.

${ }^{9}$ Every theory is interpretable in an inconsistent theory.
} 
Corollary 1 For $T 1$ with classical quantifiers and T2 with multiple quantifiers, there is a consistent theory $T$ with classical quantifiers in which $T 1$ and $T 2$ are interpretable (according to Tarski's concept of interpretability) and the quantifiers of $T$ act over the domains of the quantifiers of T2. Assuming the same premises, if $T$ is a theory with multiple quantifiers, then the quantifiers will also act over the domains of the classical quantifiers of T1.

Corollary 2 For $T 1$ and $T 2$ with multiple quantifiers, there is a consistent theory $T$ with multiple quantifiers in which $T 1$ and $T 2$ are interpretable (according to Tarski's concept of interpretability) and the quantifiers of $T$ act over the domains of the quantifiers of $T 2$.

We will now unpack the implications of the above theorem and corollaries for our present considerations. Suppose we have constructed a genuinely new formal first-order theory with at least two different existential quantifiers (including "elite" ones), and are taking into account some existing first-order theory with one classical (or special) existential quantifier, and one (or many special) universal quantifier(s). From the theorem and the corollaries it follows that there will exist a third theory with classical (or special) quantifiers, in which the first two theories are interpretable. This means that we can speak about domains containing ontologically different objects according to M-QCE in a theory which refers to objects that are common to the domains of the classical and the non-classical quantifiers. In effect, the M-QCE criterion is not then tenable, and multi-quantifier theories cannot satisfactory discern objects with different modes of being. Moreover, as we will show in the next section, there are some special theories satisfying the theorem and its corollaries, in which, for instance, the constants to which a theory is committed according to M-QCE totally change their meaning and structure. For instance, in ZFC the null set can have an additional structure consisting of an entire model of ZFC itself, and this structure cannot be detected by the classical existential quantifier or even by any of the special quantifiers. Thus, if we can describe the method of theory construction referred to in the Theorem and the Corollaries, we will have at our disposal a tool capable of showing that any quantifiers introduced to detect different ways of existence no longer have this ability. What this in turn demonstrates is that the ability to perform this ontological role does not in fact belong to "the essence" of quantification: rather, there must be tools other than quantifiers available if we are to be able to distinguish between modes of existence. In short, it is quite possible that the different modes of existence in a given theory correlate precisely with different quantifiers, but this will nevertheless be entirely coincidental: i.e. the distinction must reflect other principles, and the correlation with quantifier types will be only an effect of the latter, not their source and cause.

There are many ways in which, on the basis of this theorem, one can construct the theory T. To be sure, we could employ the sort of unification of theories described in the example given earlier, but such a union does not meet the conditions captured in the corollaries. We thus need a more specific kind of procedure: e.g. the use of a substitution method in substitutional models, as outlined in the next section.

Based on the above theorem and its corollaries, it can, moreover, be proved that there is a counterexample (in the sense described above) for every multi-quantificational theory-i.e. not only for the theory described in this section. 


\section{Intuitive Substitutional Models and Existential Quantification}

In this section we will just present the main ideas that pertain to substitutional models and substitutions. For present purposes, we shall only seek to illustrate how one can make use of substitutions and their corresponding models as these relate to "quantificational ontology".

The mathematics of mathematicians, logicians and scientists is not a pure, un-interpreted formal game. It usually involves working with intuitive models: i.e. as objects of their theories, these practitioners will adopt not only unqualified extensional "points", but also "more real" entities, such as have properties actually not formally described by the theories in question. For instance, we may adopt the "full-blooded" letters "x", "y", etc., as elements of the alphabet (or language) of a formal theory, where these are objects having a definite "shape". That shape is not precisely and formally described, ${ }^{10}$ yet such objects nevertheless serve useful functions in practice and in thinking. Any "additional properties" not described by any formula of a given language of a given theory (usually) turn out to be inessential in formal proofs, and they can (usually) be eliminated, should "formal purity" or exactness be required. Many objects which are present in our (real) thinking can be imagined or "embellished" thus. For instance, one can imagine a set as a collection of "dots" separated by some spatial distance, even though there is no definition of "spatial separation" in, say, ZFC.

We have bracketed the word "usually" in some places in the text above, because in some applications of formal theories-for instance in physics-the import of such a "decoration" is associated with the emergence of some real empirical properties; cf. (Król, 2008).

Generally speaking, the applicability of mathematics in the context of descriptions of reality and everyday life follows from some more or less intuitive transformation between mathematical objects on the one hand and real objects on the other. This is so even in cases where we employ arithmetic to count something in reality-for example, to recognize that we can present three people with four apples each when we have twelve apples altogether. Although the properties of the objects in the two transformed domains are, in principle, not the same, the "trick" works anyway. This is also the case for transformations between formal theories and intuitive models. Two basic kinds of intuitive model may be distinguished: models consisting of points and models consisting of qualified things. As we explain below, the key idea when it comes to describing intuitive models in formal terms is the notion of substitution in substitutional models.

Suppose we are presented with some formal theory T1-say, ZFC, Euclidean geometry, group theory, linear continua, etc. The elements of the model for such a theory will be some "unqualified points", or sets of such points, etc. Suppose, moreover, that every such point is substituted by an object (a model or formally described entity) belonging to some other theory T2. For example, every point on a line is substituted by a section $<0 ; 1>$, by a circle, or by a group, etc. Thus, every unqualified point becomes an object which has some additional properties that are "invisible" to the formal tools of T1, but well described in terms of T2. Such substitutions of formal theories are examples of intuitive models in

\footnotetext{
10 In general, what we think of as "languages", together with their "variables", and "symbols", imply models of an intuitive sort, just because every variable is an object of a given shape, not explicitly defined in the given language or its corresponding metalanguage. From the formal point of view, even the concept of truth in a model implies that such a model be an intuitive one, as the relations it captures only obtain thanks to certain algebras and morphisms; cf. (Rasiowa et al., 1963).
} 
mathematics. The effects of some substitutions of this kind can be easily comprehended intuitively: for instance, substitution of every point of a straight line by a straight line yields a planar surface.

There are many possible types of substitution (and corresponding substitutional models): local (finite, infinite, countable, singular etc.) substitutions, global, homogeneous or non-homogeneous substitutions, self-substitutions, etc. Examples of substitution are frequently used in mathematics, but have not received any separate and systematic treatment. There are many operations that are not, strictly speaking, substitutions, but that generate some substitutions in such a way, for example, that a given operation in category theory can be used to describe the related substitution. One can also treat as a substitution Tarski's topological version of $\mathrm{E}^{3}$, where instead of points, interiors of spheres are employed (1956). Also, the well-known fact that axioms of planimetry are true not only for points of a plane, but also for pencils of lines in the plane, may be regarded as expressing a substitution. The construction of a dual category for a given category can be expressed in the language of substitutions: in a given category, for every arrow $\mathrm{a} \rightarrow \mathrm{b}$, substitute a reverse arrow $\mathrm{b} \rightarrow \mathrm{a}$. Homomorphisms of algebraic structures may be regarded as generating substitutions, too: e.g. the reverse of surjection generates a global substitution. Note also that the construction of a model may be regarded as a substitution. This also follows from work (Rasiowa et al., 1963) showing that formalised theories and models correspond to certain algebraic structures, and that the interpretation function is a homomorphism of sorts; that, moreover, is what Lawvere (1969) writes about. Some operations performed on a given structure lead to a structure of the same type. For example, the quotient group of a given group is also a group; the inverse functor to the functor assigning a given group its quotient structure generates a substitution.

Substitutions have, however, some limitations, both formal and empirical. Formal restrictions follow, for example, from the fact that the objects introduced in many cases must be "invisible" to the formal means employed in the original theory. A contradiction immediately arises if we do not distinguish between relational and other symbols-compare the example below.

Note that although the substitution method may be applicable from a purely formal point of view, it cannot but be neutral with respect to empirical facts. For example, in the case of an entangled system of two electrons, one can formally introduce a substitution, for the electron with a spin of $+1 / 2$, of an electron with a spin of $-1 / 2$, but then one would have an entangled system of two electrons with the same spin, namely $-1 / 2$, and this is empirically impossible. Unrestricted substitutions of quantum objects by other objects may in some cases be inconsistent with Bell's inequalities, because they (i.e. unrestricted substitutions) may imply the existence of some hidden parameters in Quantum Mechanics.

Ontological limitations of substitutions concern the type of objects being substituted. However, one can substitute any objects of a certain class for any other objects of the same class. In general, semantic and syntactic substitutions may be distinguished. The latter have been widely used and find expression in some inference rules: compare, for instance, syntactical substitution rules. In principle, it is plausible to distinguish ontological categories of objects along analogous lines to syntactic categories of formal expressions. Two objects may be considered as belonging to the same ontological category if it is possible to perform their consistent substitutions in every case, and in the sense described in this paper. The notion of an object's ontological category proves helpful when exposing the ontological limitations of substitutions. At least, the following ontological categories of objects may be distinguished: syntactic objects, logical objects, mathematical objects and real objects. 
The idea of substitutions seems quite clear and easy to comprehend; nevertheless, a general formal description of it will involve some standard concepts from model theory and category theory. In the following, we present only two examples of such a description, and then apply the idea of substitution in the context of quantification, as well as to the proofs of the theorem and corollaries presented in the previous section.

The first example presented below, namely $\mathrm{ZFC}_{1} / \mathrm{ZFC}_{2}$, is a very strong counterexample to almost every possible version of M-QCE. ZFC is a theory of basic importance for those concerned with ontological commitment, while multi-quantificational theories do have models expressible in $\mathrm{ZF}(\mathrm{C})$, so it deserves some more detailed explanation.

Let us consider a substitution of $\mathrm{ZFC}_{2}$ in $\mathrm{ZFC}_{1}$ : we substitute a transitive model of $\mathrm{ZFC}_{2}$ for the empty set $\mathbf{0}$ from $\mathrm{ZFC}_{1}$, referring to such substitutions as cases of "local singular self-substitution". Obviously, the two "copies" of ZFC-i.e. $\mathrm{ZFC}_{1}$ and $\mathrm{ZFC}_{2}-$ must be written down in such a way that they have no relational or constant symbols in common: e.g. the symbols " $\in_{1}$ " and "= " in $\mathrm{ZFC}_{1}$ correspond to " $\in_{2}$ " and "= $"$ " in $\mathrm{ZFC}_{2}$. The same con-

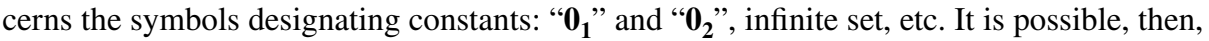
to create the third theory, $\mathrm{ZFC}_{1} / \mathrm{ZFC}_{2}$, in the following way: we write down every axiom of $\mathrm{ZFC}_{1}$, and add to these the set of renamed and supplemented axioms of $\mathrm{ZFC}_{2}$, as indicated below. For instance, the axiom of extensionality of $\mathrm{ZFC}_{2}$ now becomes: $\forall \mathbf{x}, \mathbf{y}, \mathbf{z}$. $\left[\left(\mathbf{x} \in \in_{2} \mathbf{0}_{\mathbf{1}} \wedge\right.\right.$ $\left.\left.\mathbf{y} \in_{2} \mathbf{0}_{1} \wedge \mathbf{z} \in_{2} \mathbf{0}_{1}\right) \rightarrow\left(\mathbf{x} \in \in_{2} y_{y} \equiv \mathbf{x} \in_{2} \mathbf{z} \rightarrow \mathbf{y}={ }_{2} \mathbf{z}\right)\right]$. The axiom of pairing has the form: $\forall \mathbf{x} \in$ ${ }_{2} \mathbf{0}_{1}, \forall \mathbf{y} \in \in_{2} \mathbf{0}_{1} \exists \mathbf{z} \in \mathbf{0}_{\mathbf{1}} \forall \mathbf{u} \in \mathbf{2} \mathbf{z} .\left(\mathbf{x} \in \in_{\mathbf{2}} \mathbf{z} \equiv \mathbf{u}={ }_{\mathbf{2}} \mathbf{x} \vee \mathbf{u}={ }_{\mathbf{2}} \mathbf{y}\right)$. The axiom of infinity becomes: $\exists \mathbf{z} \in_{\mathbf{2}} \mathbf{0}_{\mathbf{1}}$. $\left[\left(\mathbf{0}_{\mathbf{2}} \in \mathbf{2} \mathbf{z}\right) \wedge\left(\forall \mathbf{x} . \mathbf{x} \in_{\mathbf{2}} \mathbf{z} \rightarrow \mathbf{x} \cup\{\boldsymbol{x}\} \in_{\mathbf{2}} \mathbf{z}\right)\right]$. The remaining axioms of $\mathrm{ZFC}_{2}$ can be adapted in a similar fashion. It is also necessary to add one new axiom, the axiom of transitivity, $\forall \mathbf{x}, \mathbf{y}$. $\left(\mathbf{x} \in \in_{2} \mathbf{y} \wedge \mathbf{y} \in_{\mathbf{2}} \mathbf{0}_{\mathbf{1}}\right) \rightarrow\left(\mathbf{x} \in_{2} \mathbf{0}_{1}\right)$, in order to have every set from $\mathrm{ZFC}_{2}$ "inside" $\mathbf{0}_{\mathbf{1}} \cdot{ }^{11}$ We'll refer to the theory $\mathrm{ZFC}_{1} / \mathrm{ZFC}_{2}$ as a substitutional theory, which corresponds to a substitutional model.

Note that most of the above-mentioned formal renaming and restrictions with respect to the substitution follow from Gödel's second incompleteness theorem, which excludes the possibility of constructing a model (i.e. giving a formal description) of a theory in that theory. Note also that there is no need to use different quantifiers in a consistent description of such substitutions.

In $\mathrm{ZFC}_{1} / \mathrm{ZFC}_{2}$, unrestricted quantifiers are used in the part corresponding to $\mathrm{ZFC}_{1}$. The resulting possible mixture of sets belonging to the universe of the component $\mathrm{ZFC}$ systems has the consequence that the resulting $\mathrm{ZFC}_{1} / \mathrm{ZFC}_{2}$ will not be "a pure" substitution without some additional axioms.

One has to decide, for instance, if $\mathbf{0}_{1}={ }_{1} \mathbf{0}_{2}$, or $\forall \mathbf{x} \cdot\left[\left(\mathbf{x} \in \mathbf{2}_{\mathbf{1}}\right) \rightarrow\left(\mathbf{x}=\mathbf{1}_{\mathbf{1}} \mathbf{0 0}_{\mathbf{1}}\right)\right]$ or $\forall \mathbf{x} \cdot[\sim(\mathbf{x} \in \mathbf{2}$ $\left.\left.\mathbf{0}_{1}\right) \rightarrow\left(\begin{array}{lll}\mathbf{x}={ }_{1} & \mathbf{0} & \mathbf{0}_{2}\end{array}\right)\right]$, or introduce a global relation of identity: $\forall \mathbf{x}, \mathbf{y} \cdot(\mathbf{x}=\mathbf{y}) \equiv\left(\mathbf{x}={ }_{1} \mathbf{y} \wedge \mathbf{x}=\right.$ $\left.{ }_{2} \mathbf{y}\right) .{ }^{12}$ In cases where $\sim\left(\mathbf{0}_{1}={ }_{1} \mathbf{0}_{2}\right)$, the quantifiers will be operative over the objects of both initial systems of ZFC. However, they (i.e. the quantifiers) cannot detect all of the relevant ontological commitments of $\mathrm{ZFC}_{1} / \mathrm{ZFC}_{2}$. The objects of $\mathrm{ZFC}_{1} / \mathrm{ZFC}_{2}$ "change their nature", and this fact is "unseen" by the quantifiers.

\footnotetext{
11 This axiom is not required if we allow ourselves "to mix" objects from $\mathrm{ZFC}_{1}$ and $\mathrm{ZFC}_{2}$; however, some other axioms will then be needed.

12 Obviously, in the logic of our theory, the usual rules concerning substitutions of identical objects will be restricted to the relevant formulae. For instance, if $\left(\mathbf{x}={ }_{1} \mathbf{y}\right)$, one can substitute $\mathbf{x} / \mathbf{y}$ only in formulae built with the use of " $={ }_{1}$ " or " $\in_{1}$ ". Except for the above conditions, the pure logic remains unchanged.
} 
It is now straightforward to define, in $\mathrm{ZFC}_{1} / \mathrm{ZFC}_{2}$, the relations " $\in_{\mathbf{2}}$ " and " $=\mathbf{2}$ ", as well as the constant $\mathbf{0}_{1}$ from $\mathrm{ZFC}_{2}$. For instance, $\forall \mathbf{x}, \mathbf{y} .\left(\mathbf{x} \in \in_{2} \mathbf{y}\right)_{\mathrm{ZFC} 2} \equiv \forall \mathbf{x}, \mathbf{y} .\left(\mathbf{x} \in \in_{2} \mathbf{y}\right)_{\mathrm{ZFC} 1 / \mathrm{ZFC} 2}$ or $\mathbf{0}_{\mathbf{1 Z F C 2}}={ }_{\mathbf{2}} \mathbf{0}_{\mathbf{1}}$. Therefore $\mathrm{ZFC}_{2}\left(\right.$ and $\left.\mathrm{ZFC}_{1}\right)$ is interpretable in $\mathrm{ZFC}_{1} / \mathrm{ZFC}_{2}$.

The mutual interpretability of theories-especially ZFC-is explored in Friedman's concept calculi [8-9]. The results obtained demonstrate, in an obvious way, the fallibility of M-QCE. Moreover, examples such as the substitutions considered here-in particular that of $\mathrm{ZFC}_{1} / \mathrm{ZFC}_{2}$-also point to the inadequacy of Quine's general conception regarding the ontological commitments of theories, as extensional theories are intensionally undetermined. For instance, ZFC can speak about sets or be interpreted as, say, a recipe. Ontology appears independent of quantification; from the point of view of intensionally undetermined extensional theories, ontological properties belong to the realm of inessential "intensional decoration".

An interesting situation arises when we substitute objects defined in the language of $\mathrm{T}$ for points in a model of the theory T. We'll refer to such a substitution here as "conservative". This is what happens, for instance, when we substitute the concept of a "point" by that of a "line", in Euclidean plane geometry.

Based on the considerations above, the following remarks may be formulated (with the help of Gödel's second incompleteness theorem):

Remark 1 The relations between objects of T/T resulting from conservative self-substitution are not formally definable in terms of $\mathrm{T}$ alone.

Remark 2 One can define the relations between objects of $\mathrm{T} / \mathrm{T}$ iff the objects are not formally definable in the language of $\mathrm{T}$ alone.

Remark 3 Objects and relations of $\mathrm{T} / \mathrm{T}$ resulting from conservative substitutions are not definable both-i.e. "at the same time"-in $\mathrm{T}$ and in T/T.

Finite local substitutions can be described using many-sorted logics. Every finite manysorted logic is equivalent to a classical one-sorted logic. Therefore, finite substitutions are equivalent to a classical one-sorted theory with one type of quantifier, and one obtains the following corollary:

Corollary* For every two consistent first-order (or any-other-order) theories, $T 1$ and T2, with one type of existential quantifier, there is a consistent theory $T$ with classical (or special) quantifiers in which $T 1$ and $T 2$ are interpretable according to Tarski's concept of interpretability.

This corollary negates the M-QCE criterion in cases where theories T1 and T2 quantify over ontologically different objects, in that $\mathrm{T} 1$ and $\mathrm{T} 2$ are comparable in theory $\mathrm{T}$ with one type of quantifier. Thus, one existential quantifier (including a special one) can quantify over objects possessing different modes of existence.

The above example of a substitution involving $\mathrm{ZFC}$ is purely syntactical. There are morphisms from $\mathrm{ZFC}_{1}$ and $\mathrm{ZFC}_{2}$ to $\mathrm{ZFC}_{1} / \mathrm{ZFC}_{2}$, with such morphisms being (the functors of) interpretations in the Tarskian sense-every such theory being interpretable in the substituted version. There are also corresponding morphisms between the models of the theories, 
as explained below. ${ }^{13}$ The resulting diagrams commute. Moreover, if the substitution is consistent, the theories are also consistent. ${ }^{14}$

The syntactical description has a semantical counterpart: every model of a substitution contains a sub-model of $\mathrm{T} 1$ and $\mathrm{T} 2$. This means that the substitution will be a conservative extension of $\mathrm{T} 1$ and $\mathrm{T} 2$.

Consider now an example of global homogeneous substitution. It is obvious that the elements of a model of some theory $\mathrm{T} 1$, in which we substitute several items or copies from some theory T2, can be treated as a set (or a collection) $I$ of indexes over which one can place models (or objects) of T1. Thus, to every global homogeneous substitution corresponds a bundle of sets (or models) over the base space $\boldsymbol{I}$, and the stalk space $\mathbf{A}$, containing the objects which we substitute for every point from $\boldsymbol{I}$. Then, in the usual way, we can construct a category of bundles over $I$, i.e. $\mathbf{B n}(I)$, which is a comma category $\operatorname{Set} \downarrow \boldsymbol{I}$. Therefore, $\mathbf{B n}(I)$ is a topos. When we take into account the algebraic or topological structure of the elements of $\mathbf{A}$, we will obtain a spatial topos, or some other possible structure. A more specific description will depends on the special, individual properties of the theories that participate in the substitution, and on the type of substitution.

Take the following as an example of global homogeneous substitution as used in quantum gravity (Król, 2008): every point of $\mathbf{R}^{\mathbf{4}}$ (space-time) is replaced by an atomless Boolean algebra $\mathbf{B}_{\mathrm{x}}: \boldsymbol{i}: \boldsymbol{R}^{\boldsymbol{4}} \rightarrow\left\{\boldsymbol{B}_{x} \mid x \in \boldsymbol{R}^{4}\right\}, i \in \boldsymbol{B}_{x}$. $\mathbf{B}_{\mathrm{x}}$ is a complete atomless Boolean algebra in the countable transitive model V of ZFC, by which the forcing in V can be given. ${ }^{15}$

There are plenty of intuitive examples of global substitutions and their corresponding substitutional models. For instance, consider a Euclidean plane surface in which every point is replaced by a straight line contained in the plane. (We might use, more formally, Tarski's plane geometry, for instance (Tarski, 1959).) Assuming the axiom of choice, it is possible to choose one straight line from every pencil of lines at the given point. Then, with the use of the compactness theorem, it is possible to find such a collection of lines that are pairwise disjointed, and which is a cover of the initial surface. Another example: a line of which every point is substituted by a Euclidean three-dimensional space. Such cases also provide evidential support for Remarks 1-3 above.

Such examples falsify M-QCE. For instance, in our last example, there is no way to determine whether we will be quantifying over a point or over $\mathrm{E}^{3}$. The usual conviction that our theories speak about points is formally indiscernible from the supposition that the points have an internal structure. (From this point of view, it would be more appropriate to use categories than sets.) Note that a substitutional theory resulting from substitutions involving first-order theories will also be a first-order theory to which QCE is applicable. Many more intuitive counterexamples M-QCE are readily constructible with the use of substitutions.

Note also that one can consider series of substitutions of various types: homogeneous, nonhomogeneous, finite, infinite, countable, self-substitutions, etc. The mathematical

\footnotetext{
${ }^{13}$ Formal languages are abstract algebras; (Rasiowa et al., 1963).

14 The demonstration that the substitution $\mathrm{ZFC}_{1} / \mathrm{ZFC}_{2}$ is consistent can run along the following lines: assume that there is a first-order metatheory of ZFC, MZFC, in which a model of ZFC is described. Such a metatheory contains as its syntactical part a (renamed) counterpart of $\mathrm{ZFC}$. $\mathrm{ZFC}_{1} / \mathrm{ZFC}_{2}$ may be interpreted in MZFC. If the interpretation of some theory is consistent, then that theory will also be consistent; hence, if $\mathrm{ZFC}$ is consistent, then MZFC is consistent, and thus $\mathrm{ZFC}_{1} / \mathrm{ZFC}_{2}$ will also be consistent.

15 For an exact mathematical description of this substitution, see (Król, 2008).
} 
properties of substitutions are interesting, and can be used in the description of relations between theories usually considered quite different and independent.

After what has been said, it seems clear that the method of substitution could be applicable to our initial ontological problem-and, in particular, to the proofs of the theorem and corollaries from the previous section. Note that a singular substitution of a classical theory with only one type of existential quantifier by a theory with many such quantifiers will result in a substitution of the sort involved in the proof of Corollary 1.

Consider, finally, another variant of the argument against M-QCE that makes use of substitutions: in this case, a local self-substitution of $\mathrm{T}$ with two different existential quantifiers, that consists in replacing a constant defined in $\mathrm{T}$ by the theory itself. In effect, the same kind of existential quantifier works equally well over the domains of existential quantifiers in the resulting substitutional model. Thus, every object from $\mathrm{T}$ can possess two modes of being, and there will be no distinct correspondence between the mode of being and the type of existential quantifier involved. Moreover, the quantifiers are unable to pick up on the ontological commitments of such a theory, as the constant has an internal structure, and a description of this structure requires formal means other than quantifiers.

If the supposition that first-order theories with one existential quantifier can describe "all of mathematics" were true, then it would be feasible to think that models of our theories are all abstract objects, and that they contain only abstract objects as elements. In other words, the existential quantifier would only pertain to abstract objects. As there are at least two modes of being-abstract (formal, logical, mathematical, quantified-e.g. sets) and real (physical, material, non-mathematical-e.g. elephants)-physical reality can only be a model of our theories in a secondary sense: applications of formal theories in physics are interpretations (or intuitive models) of formal theories, rather than "exact" models of them. The formal difference between such models was indicated above, but see also the increasing role-already mentioned earlier-played by this way of treating formal models in relation to quantum gravity (Król, 2005, 2004).

Pursuing this further, our considerations imply that the quantificational criteria of existence listed in the Introduction are all problematic.

\section{Final Remarks on Existential Multi-Quantification in Ontology}

Prima facie, it seems easy to define multiple existential quantifiers within a theory. Nevertheless, as Turner writes: "Only certain quantifiers-following Ben Caplan... we'll call them elite quantifiers-matter for metaphysics" (Caplan, 2011; Turner, 2018) because, supposedly, only such quantifiers can distinguish domains of different ways of being. An analysis of the question "What makes a quantifier elite?" did not trouble us in this paper, because we believe that the proposed substitution-based approach potentially falsifies every kind of quantifier's claim to elite status in the context of ontology.

We begin the ensuing conclusions by collecting together the falsification arguments for the types of M-QCE listed in Section I, and consider some additional sub-types. The Corollary* (Section IV) provides means for constructing counter-examples to Strong QCE+and Strong M-QCE+. That is to say, by making appropriate substitutions of objects with an associated quantifier-related mode of existence for objects with some other mode of existence, it allows us to disrupt the association of quantifiers with modes of existence. Strong QCE can also be falsified by constructing appropriate substitutional extensions of a given theory so as to show that the quantifier alone cannot distinguish the ontological type of the 
object in question. With appropriate substitutions, every object from a given theory may be interpreted as having two modes of being, with no distinct correspondence between the mode of being and the type of existential quantifier involved. For example, the quantification may not distinguish whether it is over a point or a whole space, or even over the whole model (Corollaries 1 and 2). Consider strong M-QCE (Section I). If it were valid, then different modes of existence would force the use of different quantifiers: i.e. no classical theory with one existential quantifier could refer to ontologically different objects. Strong M-QCE is false because, as was demonstrated by the counterexamples presented above (cf. the counterexample associated with $\mathrm{ZF}(\mathrm{C})$ ), a theory with one existential quantifier is feasible in this instance.

There is, however, room for the weak M-QCE (cf. Section I) in two other possible variants:

Exact-weak M-QCE Ontological quantificational pluralism is possible iff it is possible to construct a theory with multiple different existential quantifiers that can, in a neutral way, distinguish the domains of these quantifiers, assuming that a given object can only have one mode of existence (and, moreover, that the same object can appear in a theory with one existential quantifier and in a theory with multiple different existential quantifiers).

Friendly-weak M-QCE One can construct a theory with many different quantifiers, and some objects can share several modes of existence (and, moreover, the same object can appear in a theory with one existential quantifier and in a theory with many different existential quantifiers).

These two variants are also possible; nevertheless, the weak version of M-QCE is false in ontological applications if the functor "iff" is used in its formulation. This is not only because there is an obvious way of constructing formal theories pertaining to different modes of existence with one existential quantifier, but also because there is a way to introduce more than two types of existential quantifier which will split the domains of the original quantifiers. The latter possibility also shows that there is no direct connection between existential quantifiers and modes of existence.

Note also that one can enlarge a theory with one existential quantifier by adding some extra quantifiers. Such an enlargement-as has been indicated already-is possible for every theory. Thus, one object can be quantified by more than one existential quantifier, and it then follows that one object can have as many modes of existence as we wish. Clearly, such multi-quantificational theories cannot decide anything in the ontology of the real world.

Consider the question of the elimination of quantifiers from theories with several different existential quantifiers. If such an elimination is possible, then there will be a model and a theory which contain ontologically different elements without any quantification, or with only one remaining type of existential (or universal) quantifier. Moreover, according to the criterion in question, the theory may have no ontological commitments; therefore, the principle of strong ontological commitment (cf. Sections I. and IV above) will be untenable, at least for complete (here Quine would agree; cf. (Quine, 1969)) or equivalent theories. For equivalent theories, it will be necessary to change the principle: a theory will be ontologically committed iff there is an equivalent theory with formulae containing one or more existential quantifiers.

Assuming the usual interrelations between quantifiers, there is also another possibility: to transform every sentence with an existential quantifier into an equivalent sentence 
with a universal quantifier. Thus, if the principle of strong ontological commitment is valid, logically equivalent sentences can generate different ontological commitmentsthat is, they can generate commitments and/or be ontologically uncommitted. This fact takes us back to the necessity of the abovementioned change to the principle.

We should also consider a model of a theory with two different existential quantifiers: for instance, as described in (Turner, 2012). One can define the domains of quantifiers in many ways; in effect, the same object can have different ways of existence, as it is possible to quantify over a given object in multiple ways.

Let us suppose that we have, "in reality", objects exhibiting two different modes of existence. It is possible to create two separate theories for these two groups of objects, each having only one (classical) existential quantifier, while at the same time, vice versa, we can create one theory with only one existential quantifier for two ontologically different domains of objects. From this it follows that existential quantification is not necessarily responsible for the detection of different modes of existence. Therefore, a more adequate criterion should be formulated: namely, that even though there is a theory with one existential quantifier which concerns ontologically different objects, the objects will count as exhibiting different modes of existence iff one can construct a second theory having at least two different existential quantifiers for these objects. (This is the criterion referred to in Section I as "the weak M-QCE".) However, in that case, such theories should be equivalent from the point of view of model theory. This point is connected with the observation that the concept of quantification is not a self-standing idea, but is rather related to other formal properties of a given theory. This point appears quite obvious, as the domain of quantifiers in a theory is defined not by the quantifiers per se, but rather by certain axioms, conditions, etc.

One can quantify over anything and everything that a given theory requires; the conviction that quantifiers have an additional ontological role to play is simply unfounded. From a purely formal point of view, the domains of quantifiers are sets or classes. We know, thanks to Gödel, that there is no one universal language possible for all mathematics. Therefore, we may have several theories postulating different objects. Thus, there are different formally possible theories dealing with ontologically different objects that nevertheless possess one type of existential quantifier. So M-QCE will not suffice to determine the ontological commitments of a theory assuming that there are different modes of existence.

As was mentioned above, there are many objects that are not picked up on by the formal apparatus of an M-QCE theory. Yet such objects can be detected by other languages and theories. Second-order properties are invisible to first-order theories (cf. the enlargements of a model of first-order arithmetic), and if such a theory is not complete, then obviously there will also be some invisible first-order objects. Such invisible objects will possess a different mode of existence from the objects of the theory, because-according to the QCE or M-QCE criterion-they are not detected by the quantifiers of the basic theory. Higher-order quantifiers are different from first-order quantifiers, so there can $b e$ theories that concern ontologically different objects that have just one existential quantifier. Therefore, M-QCE is insufficient to determine all the ontological commitments of a theory or all that is needed in the latter.

Let us suppose again that we have, "in reality", objects possessing two different modes of existence, and apply $\mathrm{ZF}(\mathrm{C})$ to these two domains (a common situation in physics.) We can now create a set-pair of objects containing an abstract and a real object, and infinitely many such "mixed" sets. The existential quantification of the theory-ZF(C)cannot indicate any difference between the modes of existence of these objects. 
Given the same assumption as in the last paragraph, we may also consider a theory with one existential quantifier that quantifies over an ontologically non-homogeneous domain of objects. There is no problem with describing such non-homogeneity in a corresponding metatheory. It is also possible to entertain a metatheory with one existential quantifier describing a universe (a model) of a theory with many different existential quantifiers. Somebody wishing to save strong M-QCE would have to prove that every metatheory describing a model of a theory with two (or more) different existential quantifiers must also be a theory with multiple relevant quantifiers. Were that to be the case, it would mean that the models of such a theory could not be described in, say, $\mathrm{ZF}(\mathrm{C}), \mathrm{NBG}$ or other classical systems for the foundations of mathematics. However, we do not know of any such theory possessing multiple different existential quantifiers. (Every known multi-quantificational theory has models expressible in $\mathrm{ZF}(\mathrm{C})$ ).

From the above, it follows that any notion of a connection between existential quantifiers and modes of existence stands in need of further justification, and that, from a formal point of view, the existence of such a connection is only one of many hypotheses. Seen in these terms, the claim that existential quantification cannot pick up on any difference between modes of existence of quantified objects, and so discloses only partially what is needed in a formal theory, seems like a rather more promising option.

Let us suppose, again, that we have constructed a theory with several different existential (even elite) quantifiers. An open question pertains to the possibility of the (mutual?) interpretability of that theory in (relation to) a theory with one existential quantifier. Should such an interpretation exist, there will be a contradiction with the strong versions of our criterion of existence. The same kind of problems pertain to the equivalence of models of the theories. One possible example of such a situation follows from the mutual isomorphism of models of categorial set theory and ZFC; cf. (Goldblatt, 1979, pp. 328-331). It is rather hard to maintain that the categorial existential quantification is "the same" as the quantification in ZF(C); cf., for instance, (Goldblatt, 1979, pp. 438-453). We are thus left with just one more option: that different ways of existential quantification will be possible in different theories-ones that nevertheless are equivalent from an ontological point of view. While it is also possible for different theories to possess different existential quantifiers without these being ontologically equivalent in the above sense, the counterexample given above will still suffice to undermine the criterion they involve. Analogously, many-sorted logic will be equivalent to the usual one-sorted logic. Speaking in general terms, there are categories in which it is impossible to interpret quantifiers. Yet does that mean that these categories have no ontological commitments whatsoever? Only according to Quine's criteria do they have no commitments.

One thing to be concluded is that in all of the cases considered, logical existential quantifiers cannot eliminate, or substitute for, sound, genuine ontological considerations, assumptions and commitments. "Carving nature at its joints", (Turner, 2013, 2018), does not work when applied at the level of that which is held to be universal.

Our most general conclusion is that analytical analyses of the variants of the quantificational criteria of existence give rise to internal technical problems which nevertheless prove to be of only secondary importance when it comes to finding solutions to the original ontological problems of major significance connected with different modes of existence. We believe that our analysis of several variants of M-QCE has shown this convincingly. It should nevertheless be clearly stated that the quantificational criteria of existence do correctly indicate that the existence of something is not a property of that something, or the same kind (mode) of property as any other qualitative features are. Ontology is, nevertheless, independent from quantification. As was stated in the 
Introduction, "something is needed" is not equivalent to "something exists", and the purposes embodied in "our needs" are not themselves the same as "ways of being". Multiple quantifiers are unable to distinguish between modes of existence, in the same way as a single quantifier is unable to do so.

Author Contribution All authors contributed to the study conception and design. Material preparation, data collection and analysis were performed by ZK and JL. All authors read and approved the final manuscript.

Funding Not applicable.

\section{Declarations}

Conflict of interest The authors declare that they have no conflict of interest.

Open Access This article is licensed under a Creative Commons Attribution 4.0 International License, which permits use, sharing, adaptation, distribution and reproduction in any medium or format, as long as you give appropriate credit to the original author(s) and the source, provide a link to the Creative Commons licence, and indicate if changes were made. The images or other third party material in this article are included in the article's Creative Commons licence, unless indicated otherwise in a credit line to the material. If material is not included in the article's Creative Commons licence and your intended use is not permitted by statutory regulation or exceeds the permitted use, you will need to obtain permission directly from the copyright holder. To view a copy of this licence, visit http://creativecommons.org/licenses/by/4.0/.

\section{References}

Balaguer, M. (1996). Towards a Nominalization of Quantum Mechanics. Mind, 105, 209-226. https://doi. org/10.1093/mind/105.418.209

Caplan, B. (2011). Ontological superpluralism. Philosophical Perspectives, 25, 79-114.

Eklund, M. (2014, November). On Quantification and Ontology. (Oxford: Oxford Handbooks). Retrieved 01 Dec. 2020 from https://www.oxfordhandbooks.com/ view/https://doi.org/10.1093/oxfordhb/97801 99935314.001.0001/oxfordhb-9780199935314-e-40

Field, H. (1980). Science without numbers: The defence of nominalism. Princeton University Press.

Friedman, H. M. (2007, 9-11 April). Interpretations, according to Tarski Nineteenth Annual Tarski Lectures: Interpretations of Set Theory in Discrete Mathematics and Informal Thinking, Lecture 1. Retrieved 14 December 2017 from http://u.osu.edu/friedman.8/foundational-adventures/downloadab le-manuscripts/

Friedman, H. M. (2009, 31 October). Concept Calculus: Much Better Than .... . Retrieved 14 December 2017 from http://u.osu.edu/friedman.8/foundational-adventures/downloadable-manuscripts/

Friedman, H. M. (2012, 02 October). Concept Calculus: Universes. Retrieved 14 December 2017 from http://u.osu.edu/friedman.8/foundational-adventures/downloadable-manuscripts/

Garbacz, A. (2019). Existencial quantifier and ontological pluralism. Axiomathes, 29, 531-540. https://doi. org/10.1007/s10516-019-09423-4

Goldblatt, R. (1979). Topoi. North-Holland Publishing Company.

Janssen-Lauret, F. (2016). Committing to an individual: Ontological commitment. Reference and Epistemology. Synthese, 193(2), 583-604.

Javier-Castellanos, A. A. (2019). Quantifier variance, ontological pluralism, and ideal languages. Philosophical Quarterly, 69(275), 277-293. https://doi.org/10.1093/pq/pqy048

Król, J. (2004). Exotic smoothness and noncommutative spaces. The Model-Theoretical Approach, Foundations of Physics, 34(5), 843-869. https://doi.org/10.1023/B:FOOP.0000022189.71690.34

Król, J. (2005, 11 June). Model Theory and the AdS/CFT correspondence. Retrieved 14 December 2019 from https://arxiv.org/abs/hep-th/0506003

Król, J. (2008). A model of space-time II the emergence of higher dimensions and field theorystring dualities. Foundations of Physics, 36(12), 1778-1800. 
Lavine, S. (2000). Quantification and ontology. Synthese, 124, 1-43. https://doi.org/10.1023/A:1005257423 415

Lawvere, F. W. (1964). An elementary theory of the category of sets. Proceedings of the National Academy of Sciences of the United States of America., 52(6), 1506-1511. https://doi.org/10.1073/pnas.52.6.1506

Lawvere, F. W. (1969). Adjointness in foundations. Dialectica, 23(3-4), 281-296. https://doi.org/10.1111/j. 1746-8361.1969.tb01194.x

Marcus, R. B. (1972). Quantification and ontology. Nous, 6, 240-250. https://doi.org/10.2307/2214772

McDaniel, K. (2009). Ways of Being. In D. Chalmers, D. Manley, \& R. Wasserman (Eds.), Metametaphysics. New Essays on the Foundations of Ontology. Oxford University Press.

Menzel, C. (1990). Actualism, ontological commitment, and possible world semantics. Synthese, 85(3), 355-389. https://doi.org/10.1007/BF00484834

Parsons, T. (1970). Various extensional notions of ontological commitment. Philosophical Studies, 21, 65-74. https://doi.org/10.1007/BF00420738

Prior, A. N. (1971). Platonism and quantification. In A. N. Prior, P. T. Geach, \& A. J. P. Kenny (Eds.), Objects of thought. Oxford University Press.

Putnam, H. (1971). Philosophy of Logic. Georg Allen \& Unwin

Quine, WVO (1948) On What There Is? Review of Metaphysics, 2, 1948/9, 21-38. (Reprinted in W. V. O. Quine. (1980). From a Logical Point of View, (pp. 1-19). Harvard University Press.)

Quine, W. V. O. (1969). Ontological relativity and other essays. Columbia University Press.

Rasiowa, H., Sikorski, R. (1963). The Mathematics of Metamathematics. Państwowe Wydawnictwo Naukowe

Schwartz, R. (1993). On 'what is said to be.' Synthese, 94(1), 43-54.

Spencer, J. (2012). Ways of being, philosophy. Compass, 7(12), 910-918. https://doi.org/10.1111/j.17479991.2012.00527.x

Tarski, A. (1956). Foundations of the geometry of solids. In A. Tarski (Ed.), Logic, semantics, metamathematics: Papers from 1923 to 1938. The Clarendon Press. ISBN 0-915144-76-X.

Tarski, A. (1959). What is elementary geometry? In J. Hintikka (Ed.), Philosophy of mathematics (pp. 164176). Oxford University Press.

Tarski, A., Mostowski, A., \& Robinson, R. M. (1968). Undecidable theories studies in logic and the foundations of mathematics. North-Holland Publishing Company.

Thibaut, G. (2013). Constructing Formal Semantics from an Ontological Perspective. The Case of SecondOrder Logics. Synthese, 191(10), 2115-2145. www.jstor.org/stable/24020002

Torza, A. (Ed.) (2015). Quantifiers, Quantifiers and Quantifiers. Themes in Logic Metaphysics and Language Synthèse Library, vol. 373 Springer. https://doi.org/10.1007/978-3-319-18362-6.

Turner, J. (2008). Ontology, Quantification, and Fundamentality. Dissertation. The State University of New Jersey. Retrieved 20th February 2018 from https://rucore.libraries.rutgers.edu/rutgers-lib /24072/pdf/1/

Turner, J. (2012). Logic and ontological pluralism. Journal of Philosophical Logic, 41(2), 419-448. https:// doi.org/10.1007/s10992-010-9167-X

Turner, J. (2013). Ontological Pluralism. Journal of Philosophy, 107(1), 5-34. https://doi.org/10.5840/jphil 201010716.

Turner, J. (2020). Ontological Pluralism. In R. Bliss, J. T. M. Miller (eds.). (Chapter 14) The Routledge Handbook of Metametaphysics, 184-195. ISBN: 978-1-138-08225-0 (hbk).

Turner, J. (2018). Recent Work on Ontological Pluralism. Retrieved 2nd January 2019 from http://www.u. arizona.edu/ jasonturner/storage/OP-Routledge.pdf

van Inwagen, P. (2003). Existence, ontological commitment, and fictional entities. In M. J. Loux \& D. W. Zimmerman (Eds.), The Oxford handbook of metaphysics. Oxford University Press.

Zbigniew Król is a philosopher who works at the Department of Philosophy and Ethics in Administration, Faculty of Administration and Social Sciences, Warsaw University of Technology, and at the Group for Philosophy and Hermeneutics of Mathematics at the Institute of Philosophy and Sociology, Polish Academy of Sciences:, Warsaw, Poland. His research concerns philosophy of mathematics, phenomenology, hermeneutics, philosophy of science, history of mathematics and science, logic, mathematics, ontology and epistemology. He is an author of three monographs: Plato and the Foundations of Modern Mathematics: the Concept of Number by Plato (in Polish, 2005), Mathematical Platonism and Hermeneutics (in Polish, 2007) and Platonism and the Development of Mathematics. Infinity and Geometry (2015). He is also a member and one of the co-founders the International Center for Formal Ontology, Faculty of Administration and Social Sciences, Warsaw University of Technology. 
Józef Lubacz is a professor at the Warsaw University of Technology. For several decades he was involved in information technology research problems. In recent years his interests are focused on philosophical issues associated with intentional creation in various areas of human activity, which lead him to fundamental ontological issues. 